\title{
Evolutionary Neural Network Learning
}

\author{
Miguel Rocha ${ }^{1}$, Paulo Cortez ${ }^{2}$, and José Neves ${ }^{1}$ \\ 1 Departamento de Informática - Universidade do Minho \\ Campus de Gualtar, 4710-057 Braga - PORTUGAL \\ 2 Departamento de Sistemas de Informação - Universidade do Minho \\ Campus de Azurém, 4800-058 Guimarães - PORTUGAL \\ mrocha@di.uminho.pt pcortez@dsi.uminho.pt jneves@di.uminho.pt
}

\begin{abstract}
Several gradient-based methods have been developed for $\mathrm{Ar}$ tificial Neural Network (ANN) training. Still, in some situations, such procedures may lead to local minima, making Evolutionary Algorithms $(E A s)$ a promising alternative. In this work, $E A s$ using direct representations are applied to several classification and regression $A N N$ learning tasks. Furthermore, EAs are also combined with local optimization, under the Lamarckian framework. Both strategies are compared with conventional training methods. The results reveal an enhanced performance by a macro-mutation based Lamarckian approach.
\end{abstract}

\section{Introduction}

In MultiLayer Perceptrons (MLPs), one of the most popular Artificial Neural Network (ANN) architectures, neurons are grouped in layers and only forward connections exist [2]. The interest in $M L P s$ was stimulated by the advent of the Backpropagation algorithm and since then several variants have been proposed, such as the RPROP [7]. Yet, these gradient-based procedures are not free from getting trapped into local minima when the error surface is rugged, being also sensitive to their parameter settings and to the network initial weights.

An alternative approach comes from the use of Evolutionary Algorithms $(E A s)$, where a number of potential solutions to a problem makes an evolving population [5,4]. $E A s$ are appealing for $A N N$ training since [8]: a global multi-point search is provided; no gradient information is required; and they are general purpose methods (the same $E A$ may be used in different types of $A N N s$ ).

Following this trend, this work aims at exploring the use of $E A s$ for $M L P$ training, when applied to classification and regression tasks.

\section{Experimental Setup}

A set of ten benchmarks was considered in this work (Table 1), endorsing two main types (column T) of problems: Classification $(C)$ and Regression $(R)$ tasks. Six real-world problems were chosen from the $U C I$ machine learning repository [3]. The $P R A$ is based on a realistic simulation of the dynamics of a robot arm. 
The artificial tasks include the famous $N$ Bit Parity [7], the $T C C$ which consists on assigning one of three colors to each block of a $3 \times 3 \times 3$ grid cube and the $S T S$, a regression task where the output is given by: $y=\sin (8 x) \times \sin (6 x)$.

Table 1. The $M L P$ learning benchmarks.

\begin{tabular}{lrrrrrr}
\hline Task T Description & C & I & H & O & W \\
\hline $6 B P$ & C Six Bit Parity & 64 & 6 & 6 & 1 & 49 \\
$T C C$ & C Three Color Cube & 27 & 3 & 8 & 3 & 59 \\
$S M R$ & C Sonar: Rocks vs Mines & 104 & 60 & 6 & 1 & 373 \\
$P I D$ & C Pima Indians Diabetes & 200 & 7 & 7 & 1 & 64 \\
$I P D$ & C Iris Plant Database & 150 & 4 & 3 & 3 & 27 \\
$W B C$ & C Wisconsin Breast Cancer & 499 & 9 & 3 & 1 & 34 \\
$S T S$ & R Sin Times Sin & 80 & 1 & 8 & 1 & 25 \\
$P R A$ & R Pumadyn Robot Arm & 128 & 8 & 8 & 1 & 81 \\
$R T S$ & R Rise Time Servomechanism & 167 & 4 & 4 & 1 & 25 \\
$P B C$ & R Prognostic Breast Cancer & 198 & 32 & 4 & 1 & 137 \\
\hline
\end{tabular}

Each problem will be modeled by a fully connected $M L P$, with one hidden layer and bias connections, being the topology given in Table 1, where columns $\mathbf{I}, \mathbf{H}$ and $\mathbf{O}$ denote the number of input, hidden and output nodes, while column W shows the number of connections. Classification tasks make use of a single binary output (if two classes are present) or one boolean value per each class. In regression problems one real-valued output encodes the dependent variable. The standard logistic activation function $\left(\frac{1}{1+e^{-x}}\right)$ was used in all classification tasks. A different strategy was adopted for the regression problems, since outputs may lie out of the co-domain $([0,1])$. Thus, the logistic function was adopted on the hidden nodes, while the output ones used shortcut connections and linear functions, to scale the range of the outputs. For all training methods, the initial weights are randomly assigned within the range $[-1 ; 1]$, being the accuracy of each MLP measured in terms of the Root Mean Squared Error (RMSE).

\section{$3 \quad$ Experiments with Evolutionary Algorithms}

In this study, direct encoding is embraced (one gene per connection weight), an alternative closer to the phenotype, allowing the definition of richer genetic operators [5]. Two mutation operators were used, namely:

- Random Mutation, which replaces one weight by a new randomly generated value, within the range $[-1,1]$; and

- Gaussian Mutation, which adds to a given gene a value taken from a gaussian distribution, with a zero mean and 0.25 standard deviation [4].

In both cases, a random number of genes is changed, between $1 \%$ to $20 \%$ of the number of $A N N$ weights. The following crossover operators were also tested: 
- Two-Point, Uniform, Arithmetical and Sum, standard EA operators [5];

- Input and Output connections, similar to a one-point crossover except that the set of input (output) connections to a node can not be disrupted [6]; and

- Hidden nodes, that combines the previous two operators; i.e., all connections to/from a hidden node can not be separated.

The $E A s$ population size was set to 30 , being the selection done by converting the fitness value (RMSE) into its ranking, and then applying a roulette wheel scheme, being used a substitution rate of $50 \%$ and the elitism value set to one. All tests were conducted using the Java language, running on a Pentium III 933 $M H z$ PC. The termination criteria was set by CPU time (100 seconds).

The results are compiled in Table 2, which shows the quality $Q_{m}$, measured by how far (in percentage) its error $\left(R M S E_{m, t}\right.$, the mean of thirty runs for the model $m$ and task $t$ ) is from the best result $\left(B_{t}\right)$, given by: $Q_{m}=100 \times$ $\left(\sum_{t \in T} \frac{R M S E_{m, t}}{B_{t}}-1\right)$ where $B_{t}=\min _{k \in M}\left(R M S E_{k, t}\right)$, and $T$ and $M$ denote the set of learning tasks and models. In the first row, only the mutation operator is applied. For the others, each operator breeds $50 \%$ of the offspring. The best performance is achieved by gaussian mutation, being no gain in using a crossover operator, thus favoring Evolutionary Programming [4]. This may be due to the permutation problem; i.e., several genomes may encode the same $A N N$ [8].

Table 2. The overall $E A$ 's results for each model $m$ ( $Q_{m}$ values).

\begin{tabular}{lcc}
\hline Crossover & Gaussian Mutation & Random Mutation \\
\hline None & $\mathbf{2 . 1 \%}$ & $148.2 \%$ \\
Two-Point & $9.8 \%$ & $143.6 \%$ \\
Uniform & $10.3 \%$ & $143.4 \%$ \\
Arithmetical & $24.3 \%$ & $146.0 \%$ \\
Sum & $74.4 \%$ & $78.3 \%$ \\
Input & $9.3 \%$ & $143.7 \%$ \\
Output & $7.8 \%$ & $143.5 \%$ \\
Hidden & $9.5 \%$ & $143.5 \%$ \\
\hline
\end{tabular}

\section{Experiments with Lamarckian Optimization}

The EAs performance can be improved by the use of the Lamarckian point of view [1]: in this work and in every generation, each individual is subject to 50 epochs of the RPROP algorithm [7], being the new weights encoded back into the chromosome (Figure 1). Two distinct Lamarckian EAs (LEAs) were tested (Table 3), with 20 individuals and one mutation operator, gaussian (column GL) or random (column RL), since the crossovers revealed poor performances. Here the comparison favors the latter macro-mutation, which may allow individuals to 


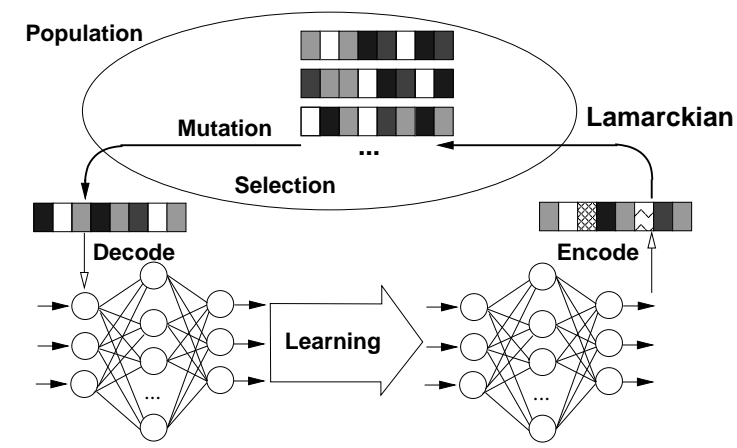

Fig. 1. An illustration of the Lamarckian strategy of inheritance.

jump between local minima, while the gaussian mutation effect may be reversed by the RPROP.

Table 3 also compares the best $E A s$ with gradient-based methods (values are presented in terms of the mean of thirty runs). The Neural Population (NP) model was added, where $20 \mathrm{MLP}$ 's will be trained via the RPROP algorithm, in order to achieve a fair comparison among population and non-population approaches. The BackPropagation (BP) is outperformed by the gaussian mutation $E A$ in four benchmarks, while the $R P R O P(R P)$ always surpasses the $E A$. The $N P$ behaves better, although the $R L$ excels all methods, stressing the importance of the random mutation and selection operators.

A temporal perspective is given in Figure 2 for the $T C C$ task, reflecting each methods' traits: the $E A$ and $B P$ show slow learning rates; the $R P$ gets the fastest convergence, but it quickly stagnates; both the random mutation $L E A$ and $N P$ reveal better long term performances, albeit the former method gains an advantage.

Table 3. Comparison between different training approaches ( $R M S E$ values).

\begin{tabular}{lcccccc}
\hline Task & EA & GL & RL & NP & RP & BP \\
\hline $6 B P$ & 0.148 & 0.078 & $\mathbf{0 . 0 3 6}$ & 0.070 & 0.243 & 0.364 \\
$T C C$ & 0.216 & 0.113 & $\mathbf{0 . 0 6 9}$ & 0.101 & 0.194 & 0.201 \\
$S M R$ & 0.153 & $\mathbf{0 . 0 0 0}$ & $\mathbf{0 . 0 0 0}$ & $\mathbf{0 . 0 0 0}$ & 0.067 & 0.045 \\
$P I D$ & 0.262 & 0.144 & $\mathbf{0 . 1 4 3}$ & 0.151 & 0.175 & 0.164 \\
$I P D$ & 0.081 & 0.045 & $\mathbf{0 . 0 3 0}$ & 0.040 & 0.064 & 0.088 \\
$W B C$ & 0.131 & $\mathbf{0 . 0 9 4}$ & $\mathbf{0 . 0 9 4}$ & 0.099 & 0.107 & 0.104 \\
$S T S$ & 0.329 & 0.095 & $\mathbf{0 . 0 7 8}$ & 0.109 & 0.095 & 0.299 \\
$P R A$ & 1.190 & 0.420 & $\mathbf{0 . 3 9 0}$ & 0.420 & 0.440 & 1.780 \\
$R T S$ & 0.571 & 0.266 & $\mathbf{0 . 2 4 2}$ & 0.254 & 0.381 & 0.523 \\
$P B C$ & 26.1 & 19.8 & $\mathbf{1 9 . 0}$ & 21.5 & 21.9 & 38.6 \\
\hline
\end{tabular}




\section{Conclusions}

Results obtained by pure $E A s$ stress the importance of the gaussian mutation and the difficulty in the design of crossover operators. Although other methods are more effective in supervised tasks, this approach can be quite useful for recurrent neural networks or reinforcement learning. For classification and regression, the experiments carried out have shown that the RPROP algorithm is the best choice when few computational resources are available. However, a better performance is achieved by the use of a Lamarckian approach, being shown that incorporating a macro-mutation is essential to obtain improved performances.

\section{References}

1. R. Belew, J. McInerney, and N. Schraudolph. Evolving Networks: Using the Genetic Algorithms with Connectionist Learning. CSE TR CS90-174, UCSD, 1990.

2. C. Bishop. Neural Networks for Pattern Recognition. Oxford Univ. Press, 1995.

3. C. Blake and C. Merz. UCI Repository of Machine Learning Databases, 1998.

4. L. J. Fogel. Intelligence Through Simulated Evolution: Forty Years of Evolutionary Programming. John Wiley, New York, 1999.

5. Z. Michalewicz. Genetic Algorithms + Data Structures $=$ Evolution Programs. Springer-Verlag, USA, third edition, 1996.

6. D. Montana and L. Davis. Training feedforward neural networks using genetic algorithms. In Proc. 11th IJCAI, pages 762-767. Morgan Kaufmann, 1989.

7. M. Riedmiller. Supervised Learning in Multilayer Perceptrons - from Backpropagation to Adaptive Learning Techniques . Comp. Stand. and Interfaces, 16, 1994.

8. X. Yao. Evolving Artificial Neural Networks. Proc. IEEE, 87(9):1423-1447, 1999.

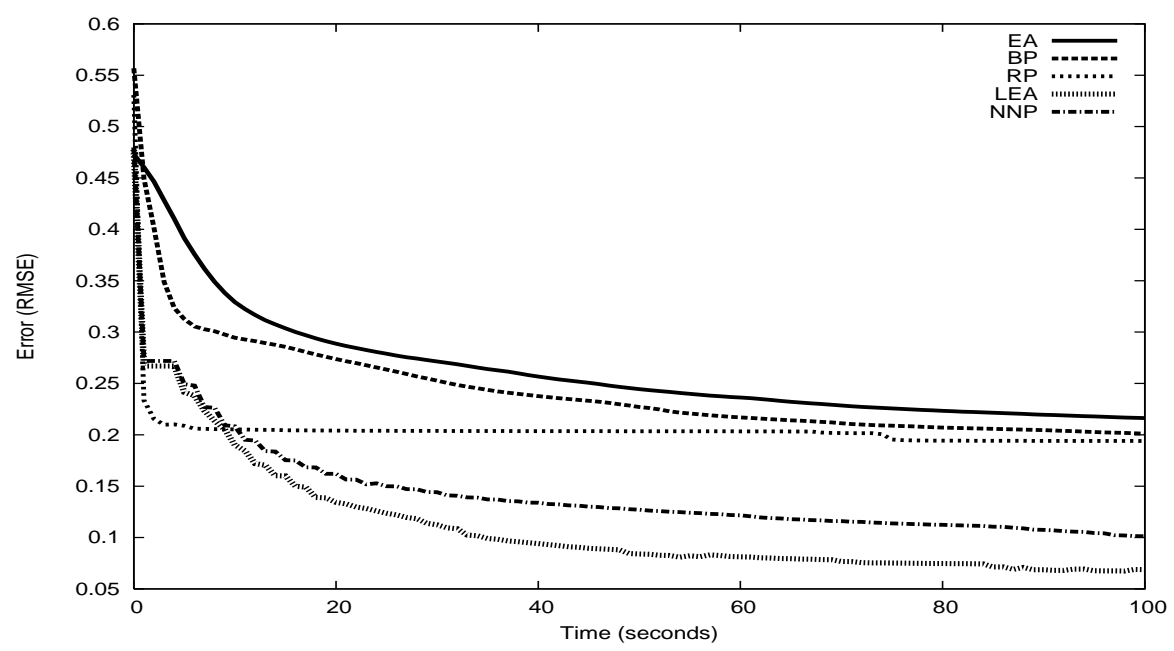

Fig. 2. The error evolution for the $T C C$ task. 